\title{
Discovery of Radio Emission from Two Anomalous X-ray Pulsars
}

\author{
V. M. Malofeev, O. I. Malov, D. A. Teplykh \\ Lebedev Physical Institute, Moscow, Russia
}

\begin{abstract}
The detection of pulsed radio emission and first results of the investigations from the anomalous X-ray pulsar (AXP) 1E 2259+586 and the AXP candidate RX J1308.6+2127 at the frequency $111 \mathrm{MHz}$ are presented.
\end{abstract}

\section{Introduction}

There is a group of five X-ray pulsars and a few candidates that have similar characteristics, and are dubbed the "anomalous X-ray pulsars" (AXPs). The first such object, 1E 2259+586, was discovered by Fahlman \& Gregory (1981) and the most recent AXP candidate, RX J1308.6+2127, by Hambaryan et al. (2002). Many attempts have been made to detect the radio emission from AXPs, because this represents an important "link" to understanding the emission mechanism of these unusual objects, which have X-ray luminosities much greater than their rate of loss of rotational kinetic energy. The modern "magnetar" model predicts the existence of radio-quiet neutron stars (e.g., Baring \& Harding 1998).

\section{Observations}

Our observations of AXP 1E 2259+586 began in 1999 Mar and our first detection of periodic radio emission was reported in 2001 Jun (Malofeev \& Malov 2001). Here we report on the detection of pulsed radio emission from the AXP candidate

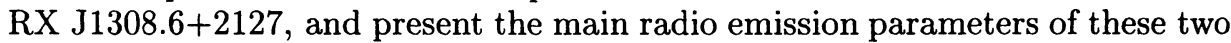
$\mathrm{X}$-ray pulsars at $111 \mathrm{MHz}$. The observations were obtained during 210 days in 1999 Mar - 2003 Apr for 1E 2259+586 and during 103 days in 2001 Dec - 2003 Apr for RX J1308.6+2127. The measurements were carried out with the Large Phased Array (LPA), a sensitive transit antenna consisting of 16384 dipoles, at an operating frequency of $110.5 \pm 1.0 \mathrm{MHz}$. All observations were made using a multichannel receiver ( 64 channels), where every channel had a width of 20 $\mathrm{kHz}$. We used a receiver time constant of $30 \mathrm{~ms}$ and a sampling interval of $25.6 \mathrm{~ms}$. The observing time was $6.2 \mathrm{~min}$ and $3.3 \mathrm{~min}$ for $1 \mathrm{E} 2259+586$ and RX J1308.6+2127, respectively. The individual pulses were recorded over a time window corresponding to one or two pulsar periods. In a single observing session, we collected 26 or 19 double period groups of pulses for each pulsar, respectively.

\section{Results}

Using the pulsar search program, we obtained a few amplitude spectra with features at frequencies matching the pulsar periods. Examples of such spectra are 

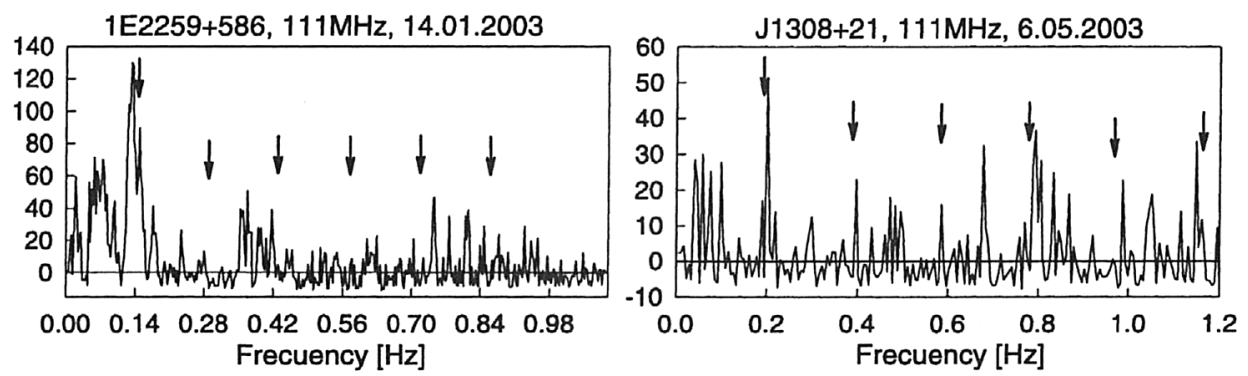

Figure 1. Amplitude spectra of two AXPs.
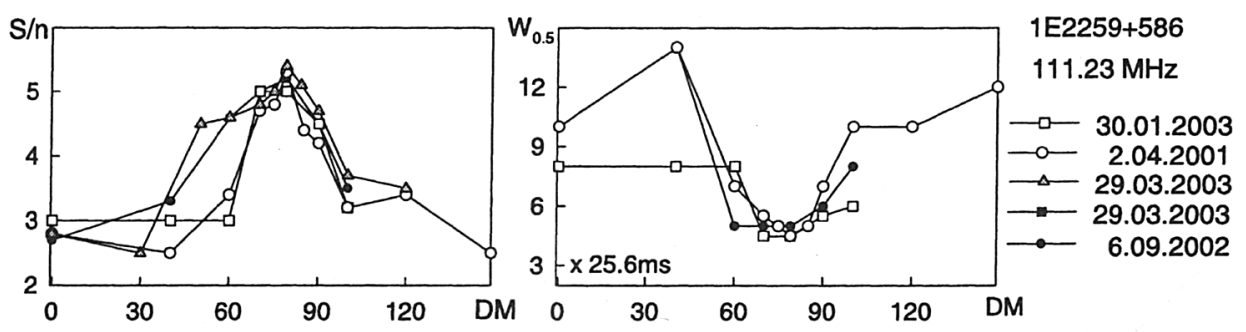

Figure 2. Signal-to-noise ratio (left) and pulse width $W_{0.5}$ (right) versus dispersion measure.

presented in Figure 1. The frequencies of the first few harmonics are shown by arrows. We computed the dispersion measure, using data from the few best days, from the time delay between the mean profiles obtained by adding the signals in a few groups of channels. These data are presented in Table 1. The search signal-to-noise ratio (left) and pulse width (right) as a function of dispersion measure are shown in Figures 2 and 3 . The distance to both objects estimated using a galactic electron density model is 3.6 and $0.25 \mathrm{kpc}$, respectively. This is in agreement with another estimates: $3.5-4.7 \mathrm{kpc}$ for $1 \mathrm{E} 2259+586$ (Gregory \& Fahlman 1980) and 0.1-1.5 kpc for RX J1308.6+2127 (Hambaryan et al. 2002).

The profile shapes (Figs. 4 and 5) are very narrow. The ratio of the pulse width at half maximum to period $W_{0.5} / P$ is $1.7 \%$ for $1 \mathrm{E} 2259+586$ and $2.7 \%$ for RX J1308.6+2127. Both profiles were obtained with an observing window
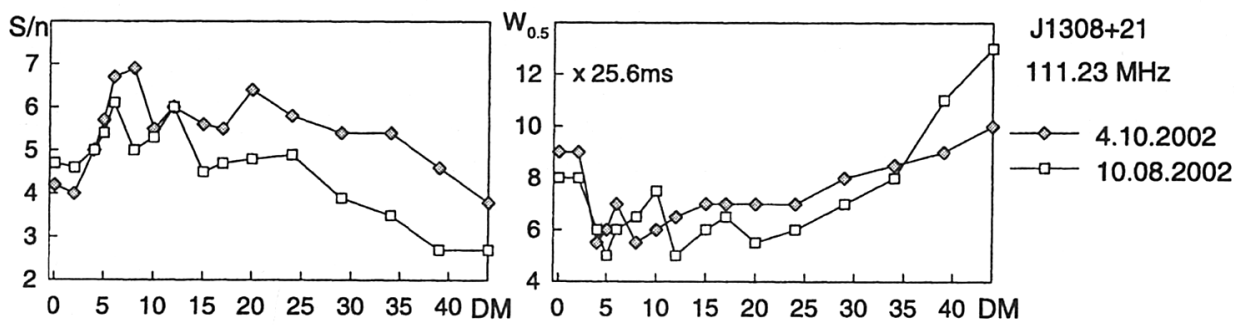

Figure 3. Signal-to-noise ratio (left) and pulse width $W_{0.5}$ (right) versus dispersion measure. 
$1 E 2259+586,111.23 \mathrm{MHz}$, sum of 12 days
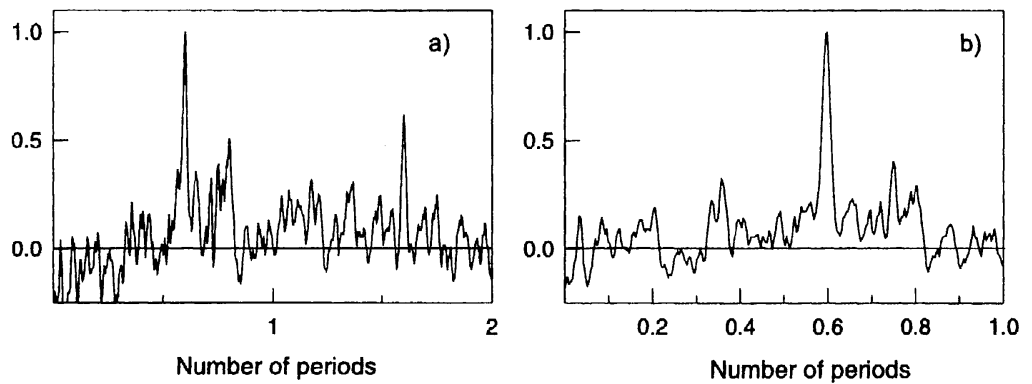

Figure 4. Mean pulse profiles obtained both by the integration of 312 double periods $P=2 P_{0}$ (a) and by the integration of 624 periods $P_{0}=6.98 s(\mathrm{~b})$.

equaling twice the apparent pulsar period (Figs. 4a and 5a). The mean profiles were obtained by the folding of data (Figs. $4 \mathrm{~b}$ and $5 \mathrm{~b}$ ). On the contrary, the X-ray profile shapes are very broad: $\sim 27 \%$ for $1 \mathrm{E} 2259+586$ and $\sim 50 \%$ for $\mathrm{RX} \mathrm{J} 1308.6+2127$. The respective signal-to-noise ratios of the mean profiles are 10 (Fig. 4b) and 14 (Fig. 5b). We could not detect the interpulse seen in the $\mathrm{X}$-ray emission of $1 \mathrm{E} 2259+586$, but very possibly we see an interpulse close to a phase of $\sim 180^{\circ}$ in the case of RX J1308.6+2127 (Fig. 5a). Indeed recent X-ray observations (Haberl et al. 2003) have shown that the real period, $P_{0}=10.31 \mathrm{~s}$, is twice that previously claimed.

On the basis of the times of arrival of 89 and 21 strong records, a timing solution has been obtained over a span of 1505 and 443 days of observations

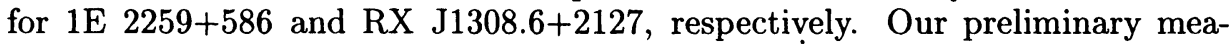
surements of periods $(P)$ and period derivatives $(\dot{P})$ are presented in Table 1 . The comparison with X-ray data shows good agreement of $P$ and $\dot{P}$ with the values published by Gavriil \& Kaspi (2002) for 1E 2259+586. Our measurements of these values for $\mathrm{RX} \mathrm{J1308.6+2127}$ are more precise than published by Hambaryan et al. (2002). We estimate the flux density of both pulsars at $111 \mathrm{MHz}$, for the 30 and 6 days, respectively, that observations were obtained which included known calibration sources. The mean flux densities are given in Table 1. We can estimate the spectral index of radio emission $(\alpha>2)$ using the upper limit on the flux density, obtained at $610 \mathrm{MHz}$ by Lorimer, Lyne \& Camilo (1998) for $1 \mathrm{E} 2259+586(S<2.3 \mathrm{mJy})$. It is also possible to estimate the luminosity for both objects, assuming a steep spectral index $\alpha \approx 2.5$ for both objects. RX J1308.6+2127 has one of the smallest value of the luminosity amongst all pulsars. We confirmed the presence of periodic radio emission from these two AXPs at even lower frequencies, 87 and $61 \mathrm{MHz}$, using the second antenna in Pushchino, the Broadband Cross Radio Telescope.

We can conclude that there is periodic radio emission from these two AXPs at low radio frequencies. This fact, together with the detection of radio pulses from SGR 1900+14 (Shitov et al. 2000), and the recent discovery by McLaughlin et al. (2003) of a new radio pulsar J1847-0130 with similar characteristics to AXPs $\left(P=6.7 \mathrm{~s}\right.$ and $\left.\dot{P}=1.3 \times 10^{-12} \mathrm{~s} \mathrm{~s}^{-1}\right)$ gives a reason to revise either the 
J1308+21, $111.23 \mathrm{MHz}$, sum of 8 days
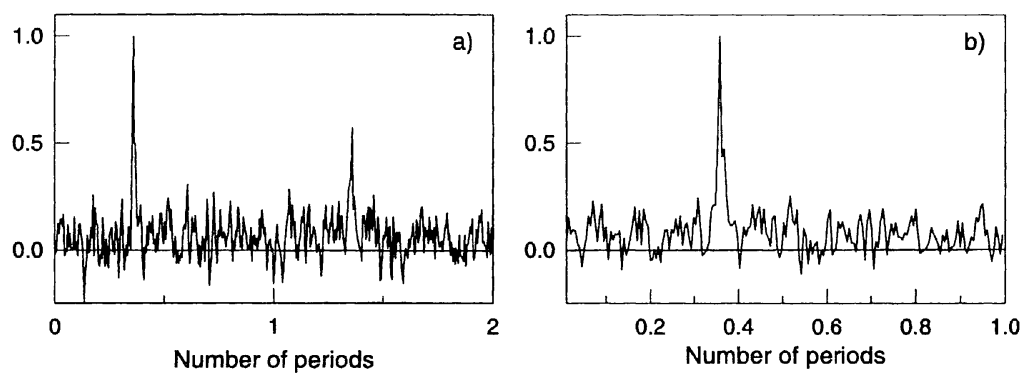

Figure 5. Mean pulse profiles obtained both by the integration of 37 double periods $P=2 P_{0}$ (a) and by the integration of 74 periods $P_{0}=5.16 s(\mathrm{~b})$.

radio emission mechanisms in the "magnetar" model, or the "magnetar" model itself.

Acknowledgments. The authors thank S. A. Tyul'bashev for help with the pulsar search program. This work was partly supported by RFBR (Pr. No. 03-02-16509, 03-02-16522), INTAS (Grant No. 00-00849), NSF (Grant No. 00-098685) and an IAU Travel Grant.

Table 1. The measured parameters of AXPs 1E 2259+586 (1) and RX J1308.6+2127 (2).

\begin{tabular}{cccccccc}
\hline PSR & $\begin{array}{c}\mathrm{P} \\
\mathrm{(s})\end{array}$ & $\begin{array}{c}\dot{P} \\
\left(10^{-13} \mathrm{~s} \mathrm{~s}^{-1}\right)\end{array}$ & $\begin{array}{c}\text { Epoch } \\
(\mathrm{MJD})\end{array}$ & $\begin{array}{c}\mathrm{DM} \\
\left(\mathrm{pc} \mathrm{cm}^{-3}\right)\end{array}$ & $\begin{array}{c}S \\
(\mathrm{mJy})\end{array}$ & $\begin{array}{c}L \\
\left(\mathrm{ergs} \mathrm{s}^{-1}\right)\end{array}$ & $\begin{array}{c}\mathrm{W}_{0.5} \\
(\mathrm{~ms})\end{array}$ \\
\hline$(1)$ & $6.97894846(6)$ & $4.87(2)$ & 51995.58 & $79(4)$ & 35 & $3 \times 10^{28}$ & $120(20)$ \\
$(2)$ & $5.15716997(2)$ & $129.96(5)$ & 51719.5 & $5.7(5)$ & 50 & $3 \times 10^{26}$ & $140(20)$ \\
\hline
\end{tabular}

\section{References}

Baring, M. G., \& Harding, A. K. 1998, ApJ, 507, L55

Gavriil, F. P., \& Kaspi, V. M. 2002, ApJ, 567, 1067

Gregory, P. C., \& Fahlman, G. G. 1980, Nature, 287, 805

Fahlman, G. G., \& Gregory P. C. 1981, Nature, 293, 202

Haberl, F. et al. 2003, A\&A, 403, L19

Hambaryan, V. et al. 2002, A\&A, 381, 98

Lorimer, D. R., Lyne, A. G., \& Camilo F. 1998, A\&A, 331, 1002

Malofeev, V. M., \& Malov, O. I. 2001, in Physics of Neutron Stars, ed. A. Koptsevich, (St Petersburg: Ioffe Physical Technical Institute), p. 31 (astro-ph/0106435)

McLaughlin, M. A. et al. 2003, ApJ, 591, L135

Shitov, Y. P., Pugachev V. D., \& Kutuzov S. M. 2000, in ASP Conf. Series, Vol. 202, Pulsar Astronomy - 2000 and Beyond, eds. M. Kramer, N. Wex, \& R. Wielebinski, (San Francisco: ASP), p. 685 